\title{
A Versatile, Low-Cost, Multiway Microfluidic Sorter for Droplets, Cells, and Embryos
}

\author{
Ramesh Utharala, Qingzong Tseng, Eileen E. M. Furlong, and Christoph A. Merten*(1) \\ European Molecular Biology Laboratory (EMBL), Genome Biology Unit, Heidelberg, Germany 69117
}

Supporting Information

ABSTRACT: Partitioning and sorting particles, including molecules, cells and organisms, is an essential prerequisite for a diverse range of applications. Here, we describe a very economical microfluidic platform (built from parts costing about U.S. $\$ 6800$ for a stand-alone system or U.S. $\$ 3700$, when mounted on an existing fluorescence microscope connected to a computer) to sort droplets, cells and embryos, based on imaging data. Valves operated by a Braille display are used to open and close microfluidic channels, enabling sorting at rates of $>2 \mathrm{~Hz}$. Furthermore, we show microfluidic 8-way sorting for the first time, facilitating the simultaneous separation and collection of objects with diverse characteristics/phenotypes. Due to the high flexibility in the size of objects that can be sorted, the low cost, and the many possibilities enabled by imaging technology, we believe that our approach nicely complements existing FACS and $\mu$ FACS technology.

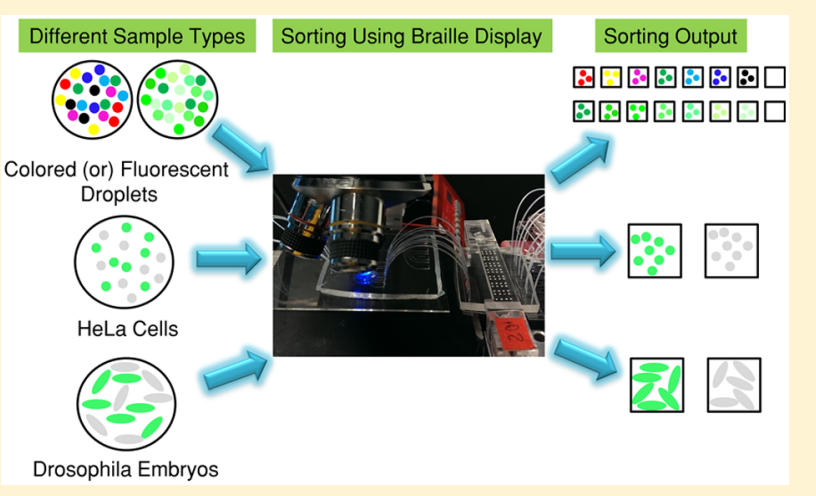

ince the development of cell sorting almost 5 decades ago, ${ }^{1,2}$ the diversity and breadth of its applications have dramatically increased, to the point where it is an essential workhorse of basic and clinical research today (a good overview on technology can be found in a previous review ${ }^{3}$ ). Modern fluorescence activated cell sorting (FACS) instruments have a throughput of several 10000 cells per second and enable the analysis of samples in many fluorescence channels simultaneously. However, these instruments are not well-suited for the sorting of very limited material (e.g., patient biopsies or other primary cells). Furthermore, the size and type of objects that can be sorted is limited, excluding, for example, aqueous microdroplets surrounded by oil, which have become a powerful format for screening applications. ${ }^{4-6}$

Microfluidic sorters nicely complement conventional benchtop equipment, as they can overcome these limitations (reviewed extensively elsewhere ${ }^{7}$. They can be based on dielectrophoresis, ${ }^{8}$ valves, ${ }^{9}$ optics, ${ }^{10}$ acoustics ${ }^{11}$ or electric stream deflec$\operatorname{tion}^{12,13}$ and even enable the use of imaging data for making sorting decisions, ${ }^{14-16}$ as long as transparent polymer chips are used. Microfluidic technology has also been integrated into commercial cell sorters such as the Cube 8 (Sysmex), the Fishman-R (On-chip Biotechnologies), the Wolf Cell Sorter (Nanocellect), and the MACSQuant Tyto (Myltenyi). These instruments enable cell sorting in different fluorescence channels, at very high throughput and with high sensitivity. However, while microfluidics has been successfully applied to the sorting of droplets, cells, and multicellular organisms, no universal device enabling the sorting of all of these objects had been described yet.

We present here a uniquely universal device, which has the further advance of enabling 8-way sorting. This not only allows sorting for fundamentally different characteristics (such as droplets of entirely different colors), but also enables the separate collection of objects showing incrementally different signal intensities (e.g., different fluorescence levels). Furthermore, the system has been assembled entirely from low cost electronic and optic modules, thus representing an easily affordable instrument for any laboratory. We believe this represents an interesting complement to existing FACS technology, especially for applications in which the throughput is not crucial.

\section{EXPERIMENTAL SETUP}

Fluidic Setup. For all experiments except the sorting of colored droplets, we used an Omano microscope (OM 118-M3) with $4 \times$ and $10 \times$ objectives. Imaging with a resolution of $656 \times$ 494 pixels was done using a Guppy F-033 B/C camera (Allied Vision Technologies) at a frame rate of $58 \mathrm{~Hz}$. Pixel values within the region of interest (ROI) were processed using LabVIEW. For sorting colored droplets, a pluggable USB 2.0 microscope with $1 \times$ magnification (Microcapture Inc.) was used and RGB values rather than pixel values were processed. The Braille unit was purchased from KGS (Extra Corp. Japan). HW30 tubing (AdTech Polymer Engineering Ltd.) with internal diameter of $0.32 \mathrm{~mm}$ and $0.23 \mathrm{~mm}$ wall thickness was used for connecting the sorting chip to the valve chip. A biopsy punch with $0.75 \mathrm{~mm}$ diameter (Harris Unicore) was used for punching inlet and outlet

Received: November 13, 2017

Accepted: April 24, 2018

Published: April 24, 2018 


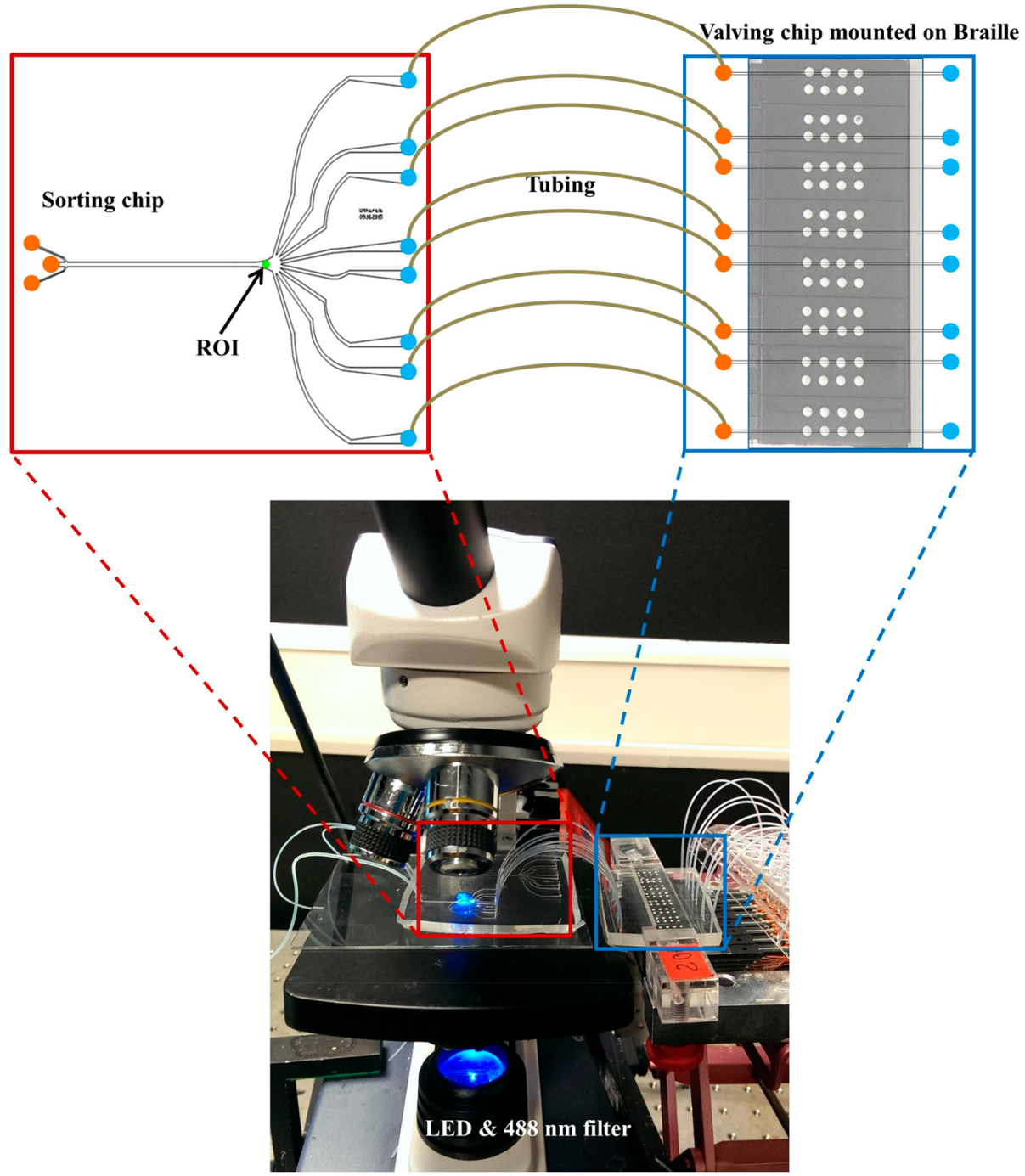

Figure 1. Microfluidic sorter. Experimental setup of the device with the sorting chip (red zoom in) and the valving chip (mounted on Braille pins; blue zoom in). The channels of the valving chip are aligned with the Braille pins below. Orange and blue circles depict the inlets and outlets of the chip, respectively.

connection holes into the PDMS chips. For exciting the fluorescent dyes, a Luxeon Rebel LED was used. The intensity of the LED was controlled by using an NI-DAQ 6009 device and LabVIEW software regulating the applied voltage $(0-5 \mathrm{~V})$. A $488 \mathrm{~nm}$ excitation filter and a 530/43 nm BrighLine $\mathrm{HC}$ emission filter (AHF Analysentechnik AG) were used for the fluorescence detection of Calcein-AM green and fluorescein. Al-4000 syringe pumps (World Precision Instruments) were used for infusing the aqueous and oil phases into the chip. For color sorting, food dye powder (European OGD Ltd.) was dissolved in water to a final concentration of $3.3 \mathrm{mM}$ for seven different colors (Table S3). $100 \mu \mathrm{M}$ fluorescein was used for droplet sorting. For all the experiments, $1 \mathrm{~mL} \mathrm{BD}$ syringes (diameter of $4.784 \mathrm{~mm}$ ) and 26 gauge $\mathrm{BD}$ needles were used.

\section{RESULTS AND DISCUSSIONS}

Working Principle. Our system is based on valves actuated by a refreshable Braille display. It comprises of 64 individually controllable pins, which can be either in an "up" or "down" position, thus enabling closing or opening of channels in polydimethylsiloxane (PDMS) chips mounted on top of the Braille unit (Figure 1). The entire system is very compact and highly cost efficient compared to pneumatic solenoid valves systems. Previous studies have applied Braille units for cell manipulation and analysis; ${ }^{17,18}$ however, until now they have never been used for cell sorting. To achieve this, we implemented two features: (i) we infused all liquids using external pumps rather than exploiting peristaltic motion of the pins. This allows much higher flow rates and ensures smooth operation without pulsing effects. (ii) We separated the sorting junction and the valves. This is necessary as the valves are just above the opaque Braille display, thus preventing imaging of this region. To overcome this limitation, we used a sorting chip connected to a second "valving chip" via polytetrafluoroethylene (PTFE) tubing. Due to the elastic nature of PDMS, the tubing and the microfluidic chip form a tight seal. Furthermore, since liquids are incompressible, closing off the channels at a distance downstream of the sorting junction is sufficient to switch liquid streams without significant delay. In consequence, the geometry and design of the sorting chip is completely independent of the valve function and can be easily modified for particular applications (e.g., based on the size of the objects to be sorted). In contrast, the more complex valving chip, which has to be precisely aligned with the Braille pins (leaving less freedom for 
A
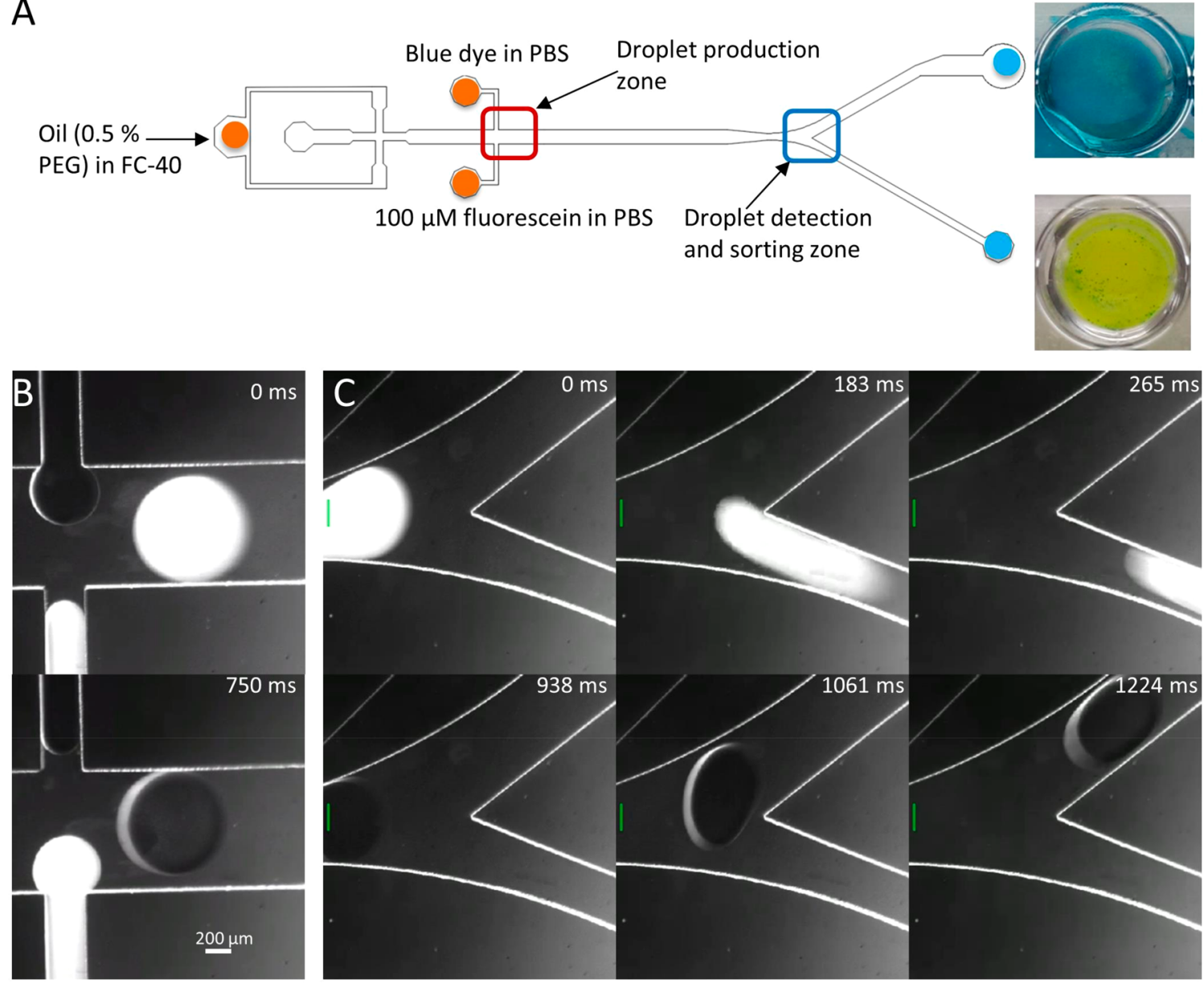

Figure 2. Two-way sorting of fluorescent droplets. (A) AutoCAD design of the 2-way sorting chip. Orange and blue circles depict the inlets and outlets of the chip, respectively. (B) Generation of alternating fluorescence positive (100 $\mu \mathrm{M}$ fluorescein) and negative droplets (blue dye in PBS) using a geometry as shown in part A. The red colored square depicts the droplet production zone and the blue colored square depicts the droplet detection and sorting zone. (C) Time-lapse imaging of 2-way droplet sorting. Fluorescence intensities are continuously measured at the region of interest (green line) and processed. A threshold is set, and every droplet exceeding the threshold is sorted to collection (below), while fluorescence negative droplets are sorted into the waste (above). Active sorting was performed by connecting the outlets of sorting chip to the inlets of the valving chip via an $8 \mathrm{~cm}$ piece of tubing (location of valves as described in Figure 1). The outlets of the collection and waste channels can be connected to wells of a 96-well plate for further analysis of the sorted fractions (as shown on the right in part A).

\section{Table 1. Sorting Statistics}

\begin{tabular}{lcccc}
\multicolumn{1}{c}{ sorting type } & $\begin{array}{c}\text { total number of sorted } \\
\text { objects }\end{array}$ & $\begin{array}{c}\text { throughput } \\
{[\mathrm{Hz}]}\end{array}$ & $\begin{array}{c}\text { percentage positives/negatives in } \\
\text { collection channel [\%] }\end{array}$ & $\begin{array}{c}\text { percentage positives/negatives in } \\
\text { waste channel [\%] }\end{array}$ \\
droplets (2-way) & 1044 & 1.36 & $\sim 100 / 0$ & $2.6 / 97.4$ \\
fluorescein droplets (2-way) 12.5 vs 15 $\mu \mathrm{M}$ & 524 & 2.10 & $\sim 100 / 0$ & $99.8 / 0.2$ \\
$\begin{array}{l}\text { fluorescein droplets (2-way) } 450 \mathrm{vs} \\
500 \mu \mathrm{M} \text { ( }\end{array}$ & 490 & 2.41 & $99.4 / 0.6$ & $0 / \sim 100$ \\
$\begin{array}{l}\text { color droplets (8-way) (see Supporting } \\
\quad \text { Data) }\end{array}$ & 143 & 0.03 & $98.9 / 1.1$ & $3.1 / 96.9$ \\
$\begin{array}{l}\text { fluorescein droplets (8-way) (See } \\
\text { Supporting Data) } \\
\text { cells }\end{array}$ & 280 & 0.13 & $99.4 / 0.7$ & $2.1 / 97.9$ \\
embryos & 2100 & 0.38 & $91.1 / 8.9$ & $19.8 / 80.2$ \\
\hline
\end{tabular}

design modifications) and whose depth is limited by the use of positive photoresist (required for preparing rounded channels that can be closed off completely), is the same for all applications, thus ensuring a high flexibility of the system.

The Braille unit itself is powered and operated using a custommade controller, enabling valve-switching within just $20 \mathrm{~ms}$ using customized LabVIEW software. Imaging is performed using a simple microscope equipped with a fire-wire camera, an additional $470 \mathrm{~nm}$ LED as light source, a $488 \mathrm{~nm}$ cleanup filter for specific blue excitation, and a $530 \mathrm{~nm}$ filter for green light emission. The LabVIEW software performs real-time processing of red, green, and blue (RGB) values of objects passing a line, which is the sorting region and sends commands to the Braille display for opening and closing the respective channels.

Droplet Sorting. To test the performance of the system, we first focused on the sorting of fluorescent droplets. We generated 

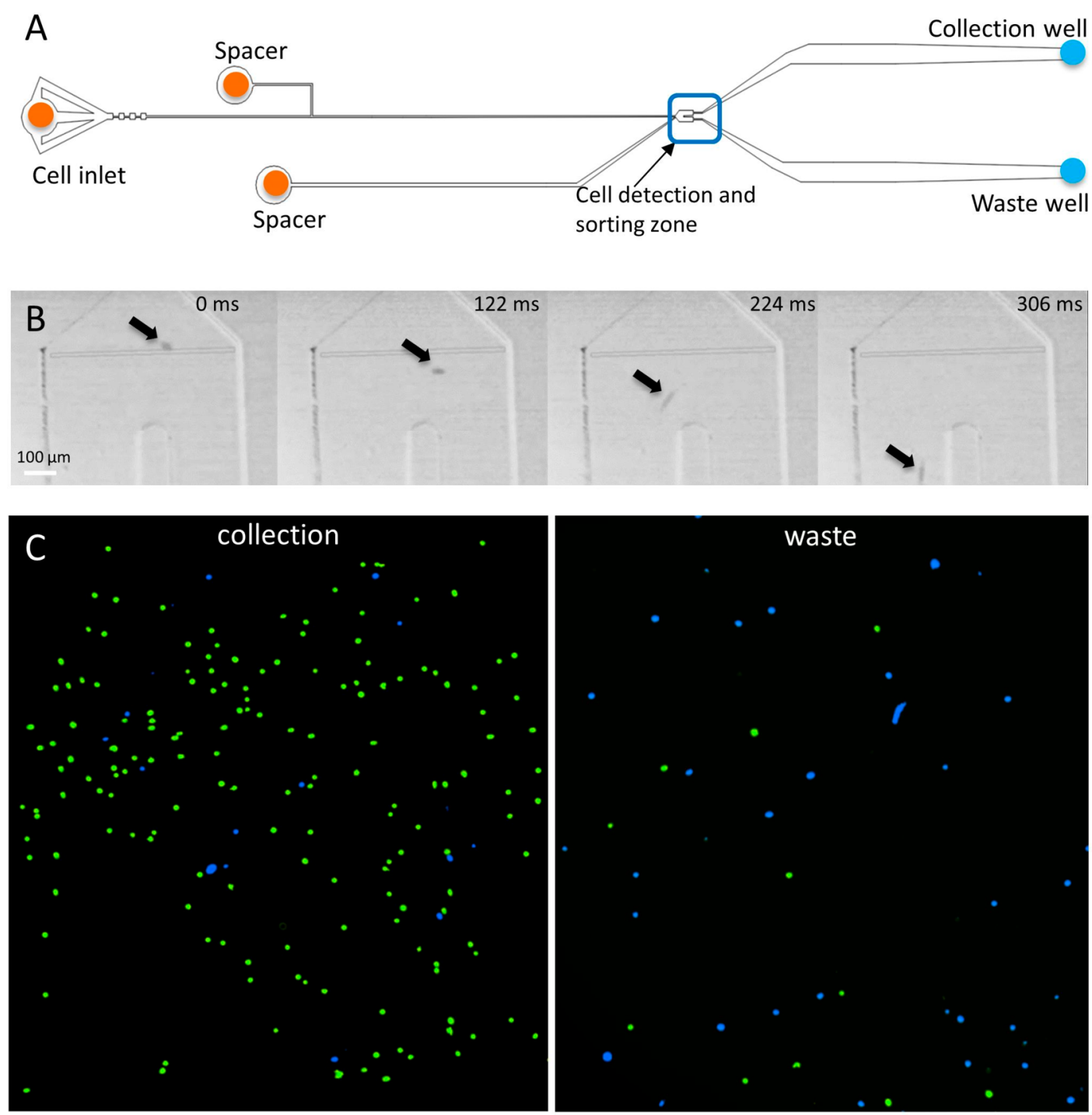

Figure 3. Sorting of green and blue stained Hybridoma cells. A 1:1 mixture of these cells was injected into the microfluidic chip and sorted according to the fluorescence in the green channel (A) AutoCAD design of the sorting chip. Orange and blue circles depict the inlets and outlets of the chip, respectively. (B) Time lapse image series of a sorting event. Active sorting was performed by connecting the outlets of sorting chip to the inlets of the valving chip via an $8 \mathrm{~cm}$ piece of tubing (location of valves as described in Figure 1). A green fluorescent cell (highlighted by black arrows) is detected and sorted into the collection channel (left) upon switching valves $300 \mathrm{~ms}$ post detection. The field of view corresponds to the section highlighted in blue in part A. (C) Fluorescence analysis of the cells in the collection (left) and waste (right) channel.

droplets using a 4-way junction geometry on the sorting chip (Figure 2A,B), in which we injected buffer from one side, $100 \mu \mathrm{M}$ fluorescein from the other side, and oil perpendicularly. This resulted in the stable generation of alternating fluorescein (positive) and buffer (negative) droplets (Movie S1). Using a $470 \mathrm{~nm}$ LED light source and specific excitation/emission filters (see optical setup in Figure 1) the fluorescence of the droplets could easily be detected, enabling 2-way sorting with more than $99 \%$ efficiency on average (Figure 2C, Movie S2 and Table 1). To demonstrate the sensitivity of the system, we further performed sorting of droplets showing fluorescence intensities differing by as little as $20 \%$ at up to $2.4 \mathrm{~Hz}$ (Movies S3 and S4). The minimum fluorescein concentration that could be detected was 5 $\mu \mathrm{M}$ using the highest electronic gain. In this setting, saturation occurs at concentrations exceeding $250 \mu \mathrm{M}$ (Figure S6). This sensitivity is clearly below that of a FACS machine. However, our platform is based on imaging data (in principle also allowing to sort for colors, shapes, and other phenotypes) and could be upgraded with more expensive cameras or better optics (e.g, of an existing microscope), in case higher sensitivity is required. Using a Braille display with a total of 64-pins further allowed 8way sorting of droplets with different fluorescence intensities or differently colored droplets and also enabled the transfer of individually sorted droplets into the wells of a 96-well plate (Supporting Data, Movies S5-S7 and Figure S8).

Cell Sorting. To show the versatility of our device, we also focused on the sorting of cells based on fluorescence, which is a standard application in cell biology. We mixed 2 populations of a Hybridoma cell line, one stained with the viability dye CalceinAM green and another with Calcein-AM blue. Starting with a 1:1 ratio, the sample was injected into a 2-way microfluidic sorting chip (Figure 3A), excited at $488 \mathrm{~nm}$, and imaged at 10-fold magnification. A threshold was set to gate only on the CalceinAM green positive cells (Figure 3B), such that whenever a green cell passed the detection point, the Braille pin opened the green channel momentarily for $300 \mathrm{~ms}$, while in absence of any green 

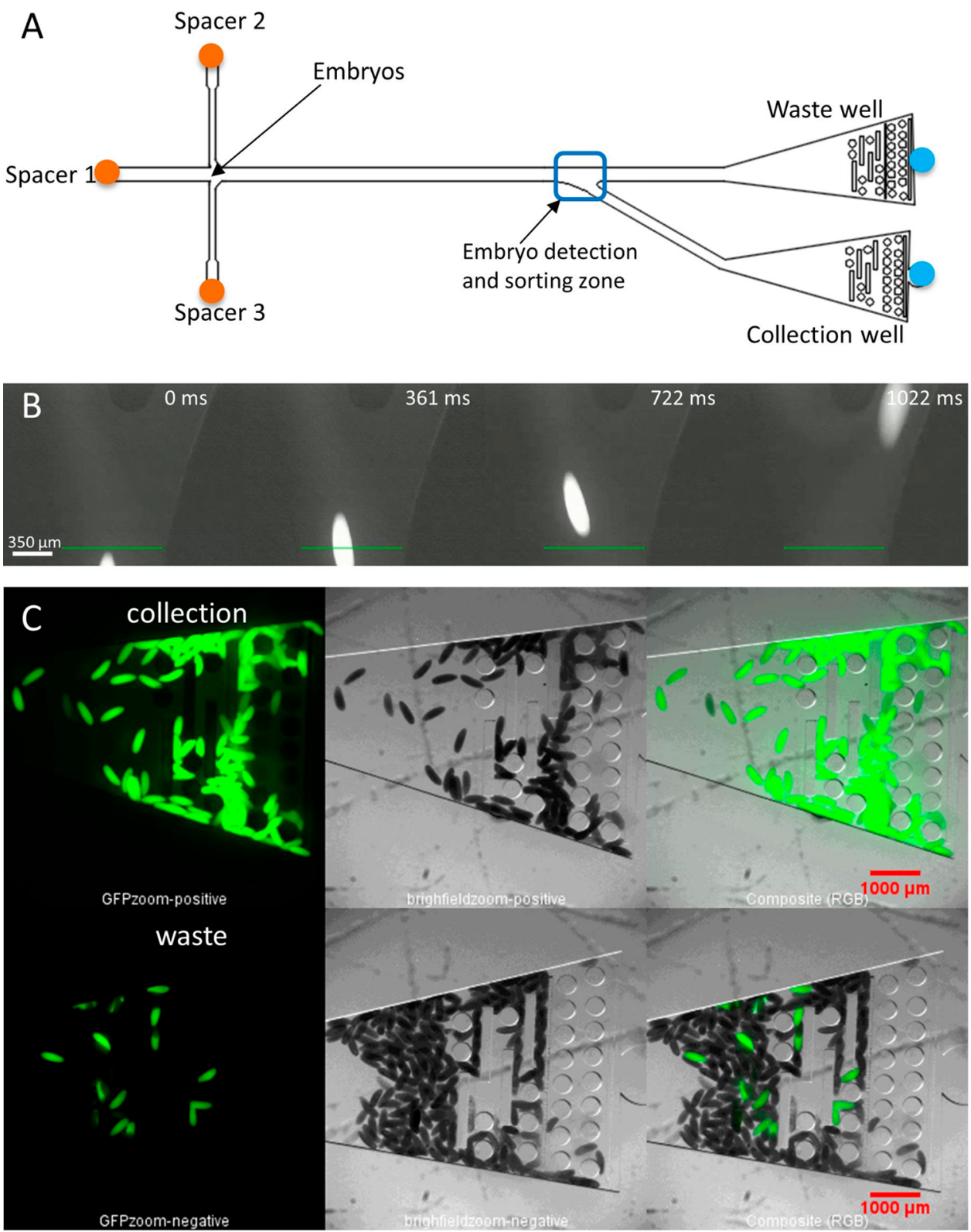

Figure 4. Sorting of Drosophila embryos (A) AutoCAD design of the microfluidic chip. The collection channel was used for collecting fluoresceinstained embryos while unstained embryos were sent to the waste. Orange and blue circles depict the inlets and outlets of the chip, respectively. (B) Time lapse-imaging of a sorting event within the field of view highlighted by a blue rectangle in part A. Active sorting was performed by connecting the outlets of sorting chip to the inlets of the valving chip via an $8 \mathrm{~cm}$ piece of tubing (location of valves as described in Figure 1). A green fluorescent embryo was sorted into the collection channel upon switching valves $750 \mathrm{~ms}$ post detection (measuring the pixel intensity across the green line). (C) Fluorescence analysis of the embryos in the collection and waste wells.

signal all liquid was diverted to the waste channel. A total of 2100 cells were sorted at a rate of $0.38 \mathrm{~Hz}$ (Movie S8) and subsequently analyzed by fluorescence microscopy (Figure 3C). This revealed a sorting efficiency of $>91 \%$ (see detailed results in Table 1). Staining sorted cells additionally with Calcein-violet (cell viability dye) further allowed determination of cell viability following sorting. Cells showing a double positive green/violet stain indicated that they were alive prior to the sort and after the sort. Cells being positive in the green channel only indicated that they had died during the sorting procedure. The obtained results (Figure S7) clearly indicated that the cell viability is not significantly affected by the sorting procedure (>99\%). To work under sterile conditions, PDMS chips can be sterilized in many different ways as shown previously. ${ }^{19}$ In our hands, flushing the chip with ethanol was sufficient and also allowed for using the same device at least 10 consecutive times.

Drosophila Embryo Sorting. Next, we focused on the sorting of bigger objects such as Drosophila embryos, which are a powerful model system in developmental biology. ${ }^{20,21}$ These embryos are approximately $520 \mu \mathrm{m} \times 180 \mu \mathrm{m} \times 180 \mu \mathrm{m}$ in size, for which reason we designed a deeper sorting chip (Figure 4A), while still using the same valving chip as before. Starting with a mixed population of fluorescein stained embryos (positives) and unstained embryos (in a ratio of $1: 1$ ), the samples were injected into the microfluidic device and imaged at 4-fold magnification. In order to implement sorting, a threshold was set so that the auto fluorescence of the negative embryos did not trigger switching of the valves, while fluorescein-stained positive 
embryos could be specifically sorted (Figure 4B). Subsequent to the sort, the collected embryos were analyzed in the collection and waste wells (Figure 4C).

A total of 282 embryos were sorted at a rate of $0.13 \mathrm{~Hz}$ (Movie S9) and subsequently the deep channels were imaged and analyzed to determine the sorting efficiency. There were minor sorting errors observed during the sorting experiment that lead to 1 false positive (out of 106 in the collection channel) and 14 false negatives (out of 176 in the waste channel) as shown in Table 1.

\section{CONCLUSION}

We have developed a highly flexible microfluidic platform capable of sorting objects of various sizes, ranging from a few to several hundred micrometers. Using droplets, cells, and multicellular organisms, we have successfully demonstrated sorting based on both bright light and fluorescence imaging at a maximal rate of $\sim 2.4 \mathrm{~Hz}$. Noteworthy, the response time of our sorting system (Supplemental text and Movies S11 and S12) is just 20 ms, thus allowing a maximum theoretical throughput of $50 \mathrm{~Hz}$ for evenly spaced objects such as droplets. Without doubt, professional FACS sorters enable much higher throughput, but they come at significantly higher cost and cannot process droplets or low cell numbers.

Furthermore, our system could be used for high-content imaging based sorting, which is so far not possible using conventional FACS instruments. For this kind of application, the throughput-limiting factor is image acquisition and analysis, rather than the fluidic control. In line with this, our design revealed that flexible and expandable PDMS chips tolerate the closure of all valves (while continuously injecting liquid) for time periods of up to $2 \mathrm{~s}$, thus enabling objects such as Drosophila embryos to be completely stopped during image acquisition (Movie S10).

Independently of any specific sorting application, the low cost of the system should allow for widespread use in biology. The price of the components used in the sorting platform shown here is about U.S. $\$ 6800$ for a custom-built stand-alone prototype and mass produced devices could probably be manufactured much cheaper. Furthermore, our microfluidic sorter could be mounted on existing microscopes and imaging platforms, thus roughly saving another U.S. \$3100, while enabling more complex optics (Table S1). Inexpensive fluidic switches can also be realized using solenoid valves. ${ }^{22}$ However, compared to these, our approach has two main advantages: First of all, the Braille valves are aligned with the channels and thus produce minimal or no dead volumes. In contrast, external solenoid valves typically have dead volumes of several microliters which are not tolerable when working with precious samples or limited material such as primary cells. Second, the Braille system is very compact and can be fully integrated into existing microscope platforms.

Taken together, we believe that our microfluidic sorter nicely complements existing FACS and $\mu$ FACS technologies, with particular benefits for the sorting of rare cells, complex specimens (such as droplets or large multicellular organisms), or samples that should be sorted based on imaging.

\section{ASSOCIATED CONTENT}

\section{(S Supporting Information}

The Supporting Information is available free of charge on the ACS Publications website at DOI: 10.1021/acs.analchem.7b04689.
Section I describes the building of a braille sorter, which includes the relevant tables (pricing information, selection criteria for sorting color and fluorescent droplets), valving technology, and additional information on custom-built parts. Section II shows the description on movies M1M12. Section III describes the supporting data, which includes the preparation of microfluidic chips, sorting conditions for droplets and biological specimen, and multichannel sorting of droplets. (PDF) (ZIP) (ZIP)

\section{AUTHOR INFORMATION}

\section{Corresponding Author}

*E-mail: merten@embl.de.

ORCID ${ }^{\circ}$

Christoph A. Merten: 0000-0001-8790-4659

\section{Notes}

The authors declare no competing financial interest.

\section{ACKNOWLEDGMENTS}

The authors would like to acknowledge Nachiket Shembekar and Lucia Ciglar and Raquel Marco-Ferreres for helping with the cell culture and Drosophila embryo collection, respectively. The authors would also like to thank the mechanical and electrical workshop at EMBL, Heidelberg, for their support and Martine Ballinger for critical reading of the manuscript. This work was funded by EMBL intramural funds.

\section{REFERENCES}

(1) Fulwyler, M. J. Science 1965, 150, 910-911.

(2) Herzenberg, L. A.; Sweet, R. G.; Herzenberg, L. A. Sci. Am. 1976, 234, 108-117.

(3) Herzenberg, L. A.; Parks, D.; Sahaf, B.; Perez, O.; Roederer, M. Clin. Chem. 2002, 48, 1819-1827.

(4) Joensson, H. N.; Andersson Svahn, H. Angew. Chem., Int. Ed. 2012, 51, 12176-12192.

(5) Dressler, O. J.; Maceiczyk, R. M.; Chang, S. I.; deMello, A. J. J. Biomol. Screening 2014, 19, 483-496.

(6) Shembekar, N.; Chaipan, C.; Utharala, R.; Merten, C. A. Lab Chip 2016, 16, 1314-1331.

(7) Wyatt Shields Iv, C.; Reyes, C. D.; Lopez, G. P. Lab Chip 2015, 15, $1230-1249$.

(8) Hu, X.; Bessette, P. H.; Qian, J.; Meinhart, C. D.; Daugherty, P. S.; Soh, H. T. Proc. Natl. Acad. Sci. U. S. A. 2005, 102, 15757-15761.

(9) Fu, A. Y.; Spence, C.; Scherer, A.; Arnold, F. H.; Quake, S. R. Nat. Biotechnol. 1999, 17, 1109-1111.

(10) Landry, Z. C.; Giovanonni, S. J.; Quake, S. R.; Blainey, P. C. Methods Enzymol. 2013, 531, 61-90.

(11) Schmid, L.; Weitz, D. A.; Franke, T. Lab Chip 2014, 14, 37103718.

(12) Dittrich, P. S.; Schwille, P. Anal. Chem. 2003, 75, 5767-5774.

(13) Chen, C. H.; Cho, S. H.; Chiang, H. I.; Tsai, F.; Zhang, K.; Lo, Y. H. Anal. Chem. 2011, 83, 7269-7275.

(14) Chung, K. H.; Crane, M. M.; Lu, H. Nat. Methods 2008, 5, 637643.

(15) Zang, E.; Brandes, S.; Tovar, M.; Martin, K.; Mech, F.; Horbert, P.; Henkel, T.; Figge, M. T.; Roth, M. Lab Chip 2013, 13, 3707-3713.

(16) Kovac, J. R.; Voldman, J. Anal. Chem. 2007, 79, 9321-9330.

(17) Gu, W.; Zhu, X.; Futai, N.; Cho, B. S.; Takayama, S. Proc. Natl. Acad. Sci. U. S. A. 2004, 101, 15861-15866.

(18) Tung, Y. C.; Torisawa, Y. S.; Futai, N.; Takayama, S. Lab Chip 2007, 7, 1497-1503.

(19) Skaalure, S. C.; Oppegard, S. C.; Eddington, D. T. J. Undergrad. Research 2017, 2, 1-4.

(20) Spitz, F.; Furlong, E. E. M. Nat. Rev. Genet. 2012, 13, 613-626. 
(21) Furlong, E. E. M.; Profitt, D.; Scott, M. P. Nat. Biotechnol. 2001, 19, 153-156.

(22) Islam, M. Z.; McMullin, J. N.; Tsui, Y. Y. Cytometry, Part A 2011, $79 A, 361-367$. 